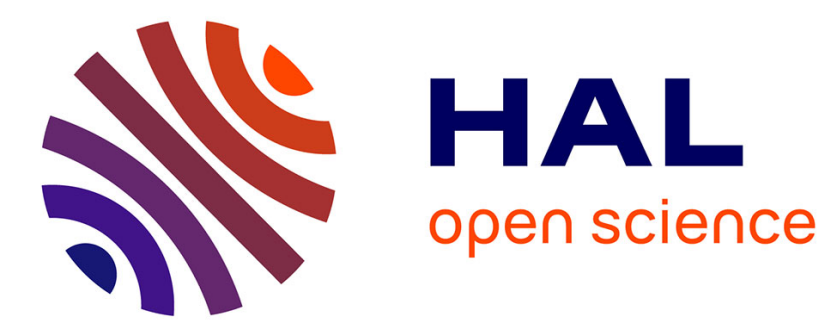

\title{
Le processus collectif de création d'un feuilleton télévisé
}

Muriel Mille

\section{To cite this version:}

Muriel Mille. Le processus collectif de création d'un feuilleton télévisé: Une division du travail d'auteurs. Sociétés contemporaines, 2016, 1 (101), pp.91-114. 10.3917/soco.101.0091 . halshs02003588

\section{HAL Id: halshs-02003588 \\ https://shs.hal.science/halshs-02003588}

Submitted on 1 Feb 2019

HAL is a multi-disciplinary open access archive for the deposit and dissemination of scientific research documents, whether they are published or not. The documents may come from teaching and research institutions in France or abroad, or from public or private research centers.
L'archive ouverte pluridisciplinaire HAL, est destinée au dépôt et à la diffusion de documents scientifiques de niveau recherche, publiés ou non, émanant des établissements d'enseignement et de recherche français ou étrangers, des laboratoires publics ou privés. 


\section{LE PROCESSUS COLLECTIF DE CRÉATION D'UN FEUILLETON TÉLÉVISÉ}

Une division du travail d'auteurs

Muriel Mille

Presses de Sciences Po | «Sociétés contemporaines »

2016/1 N 101 | pages 91 à 114

ISSN 1150-1944

ISBN 9782724634716

Article disponible en ligne à l'adresse :

https://www.cairn.info/revue-societes-contemporaines-2016-1-page-91.htm

Distribution électronique Cairn.info pour Presses de Sciences Po.

(C) Presses de Sciences Po. Tous droits réservés pour tous pays.

La reproduction ou représentation de cet article, notamment par photocopie, n'est autorisée que dans les limites des conditions générales d'utilisation du site ou, le cas échéant, des conditions générales de la licence souscrite par votre établissement. Toute autre reproduction ou représentation, en tout ou partie, sous quelque forme et de quelque manière que ce soit, est interdite sauf accord préalable et écrit de l'éditeur, en dehors des cas prévus par la législation en vigueur en France. Il est précisé que son stockage dans une base de données est également interdit. 
Muriel MILLE

\section{Le processus collectif de création d'un feuilleton télévisé \\ Une division du travail d'auteurs}

La production d'un feuilleton télévisé repose sur une organisation du travail fortement rationalisée et divisée. Le processus collectif de création est source de tensions autour des mandats des différents intervenants. Le caractère industriel de la création questionne la place des auteurs et renverse une partie des hiérarchies professionnelles existant dans le monde audiovisuel. Cet article examine les formes de coopération et de concurrence créées par cette division du travail entre les différents intervenants du processus de production. La chaîne de fabrication morcelle le travail d'écriture, et rationalise la division du travail pour produire un flux d'épisodes. Cette division redéfinit les rôles des scénaristes et leurs spécialités. Cependant malgré l'importance des contraintes pesant sur les auteurs, l'industrialisation de la fabrication leur donne aussi un pouvoir important sur le contenu des épisodes.

\section{The Production of Fiction as an Assembly-line. Division of Labor in the Making of a Daytime Soap Opera}

The production of a television series is based on a deeply rationalized and divided organization of labor. The collective process of creation reveals dissensions concerning the mandates of the various workers. The industrialized manufacturing of TV fiction questions who holds the authorship on the product and thus professional hierarchies in this art world. This article shows the contradictions among the cooperating partners, each of them following rival professional logics, and trying to reinterpret and make theirs the production constraints in their creative work. The rationalization of the production leads to conflict of jurisdictions between the various professionals involved, especially the scriptwriters specialized in the storylines and the ones writing the dialogues, and also between the writers and the people responsible of the shooting.

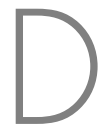

evenues le format de fiction le plus consommé et le plus diffusé à la télévision, les séries télévisées se sont imposées comme un objet d'étude en sciences sociales (Sepulchre, Maigret, 2011 ; Bacqué et al., 2014 ; Mathieu, 2013). Ces travaux, portant fréquemment sur des séries en provenance des États-Unis, visent principalement à analyser les représentations du monde social portées par ces biens culturels. Les modalités de fabrication des séries tout comme les professionnels impliqués ont cependant été peu étudiés. Or la production de séries françaises se développe, représentant en 2013, 86,8\% des œuvres audiovisuelles de fiction produites (CNC, 2014): le volume horaire des séries françaises est passé de 599 heures en 2009 à 678 heures en 2013, ce qui s'accompagne de 
formes de rationalisation de la production, et en particulier d'une intensification de la division du travail d'écriture. La production des séries «associe le travail d'auteurs de plus en plus nombreux et de plus en plus spécialisés (bible, synopsis, dialogues) », selon l'Observatoire permanent des contrats audiovisuels ${ }^{1}$ (SACD, 2012). Ce constat s'accompagne de craintes face aux «aspects sauvages de l'industrialisation » et à une possible détérioration des conditions de travail des scénaristes (SACD, 2012). Dans ce cadre, il semble important d'appréhender comment ce caractère «industriel » de la fabrication se traduit dans les modalités concrètes de la production.

Le caractère collectif et industrialisé de la fabrication des fictions (et séries) télévisées a été largement constaté pour les États-Unis (Caldwell, 2008; Banks et al., 2009), et ce depuis la fin des années 1970 et le succès croissant de ce format (Gitlin, 2000). En France, c'est au cours des années 1980 que la production de fiction télévisée s'industrialise (Pasquier, 1995). Cependant les modalités de la division du travail dans ces modes de production rationalisés ont été peu souvent prises comme objet d'analyse, aux États-Unis comme en France. Les premiers travaux américains sur le sujet se centrent davantage sur la figure du producteur (Cantor, 1971; Newcomb, Alley, 1983), ou sur les carrières des scénaristes dans ces organisations par projet (Bielby, Bielby, 1996). En France, ce sont également les professions de scénaristes (Pasquier, 1995) et de producteurs qui ont été davantage étudiées (Dagnaud, 2006 ; Brigaud-Robert, 2011). Plus récemment, les critiques et analyses des séries télévisées américaines ont mis en avant le rôle du showrunner, un scénariste porteur du projet qui en supervise l'exécution de l'écriture à la réalisation. Il est souvent présenté comme «l'auteur » de la série ${ }^{2}$, soit un équivalent de la figure de l'artiste créateur (Newman and Levine, 2012), les détails de la chaîne de coopération dans lequel il s'insère restent ainsi peu étudiés.

Le travail de production d'une série télévisée se présente en effet comme une activité éminemment collective impliquant de très nombreux intervenants, comme c'est le cas dans les mondes de l'art (Becker, 1988, p. 39), même si le plus souvent l'activité ne s'organise pas autour d'un seul individu auquel est attribuée la responsabilité de l'œuvre. La division du travail ne repose pas sur la différenciation claire entre un artiste et du personnel de renfort. Plusieurs catégories de personnel peuvent revendiquer l'autorité sur l'œuvre, alors même

1/ Un comité de la Société des auteurs compositeurs dramatique (SACD) chargée de la gestion des droits d'auteurs et de diffusion des œuvres.

2/ Les analyses mettent en avant des showrunners réussissant à contourner les contraintes de la production industrielle pour réaliser une « œuvre» (Esquenazi, 2010). 
qu'ils relèvent de professions artistiques ${ }^{3}$ valorisant la figure de l'artiste-créateur (Bourdieu, 1992). Le caractère industriel de la fabrication transforme alors la manière dont ces différents professionnels définissent leurs tâches, créant des concurrences entre eux. Il apparaît nécessaire d'analyser les modalités concrètes de la division du travail et les relations de concurrence ou de coopération entre ces différents professionnels (Hughes, 1996, 128). La notion de « territoire professionnel ${ }^{4} \gg$ de Andrew Abbott sera utilisée pour comprendre comment cette concurrence interprofessionnelle se noue dans le contenu du travail lui-même (Abbott, 1988, 20). Il s'agira d'appréhender les capacités inégales des professionnels à protéger leur « territoire », c'est-à-dire l'ensemble des tâches qu'une profession contrôle dans la division du travail ${ }^{5}$ (Abbott, 1988, p. 64) en se penchant sur un exemple singulier d'organisation du travail. Les modalités de cette division du travail sont analysées à partir d'une enquête menée sur le cas « limite» que constitue Plus Belle La Vie, feuilleton diffusé sur France 3 depuis le 30 août 2004, à la production fortement rationalisée et situé au pôle commercial du champ de production culturelle (Bourdieu, 1992).

Plus Belle La Vie (PBLV), feuilleton diffusé 5 soirs par semaine à $20 \mathrm{~h} 15$, se singularise face au reste de la production audiovisuelle par sa longévité avec plus de dix ans d'existence et de 2500 épisodes. II est produit par Telfrance Séries et Rendez-vous Production en collaboration avec les équipes techniques de France 3 Provence-Alpes-Côtes-d'Azur. Avec un budget de 35,7 millions d'euros en 2012 et 117 heures de diffusion, PBLV représentait 25,7\% du montant et $50,4 \%$ du volume horaire de fiction commandée par France 3 (CNC, 2014). Telfrance Séries et Rendez-vous Production, qui produisent presque exclusivement le feuilleton, constituent ainsi les principales sociétés productrices de fiction en $2012^{\circ}$. La fabrication des 5 épisodes d'une semaine de diffusion implique une quinzaine de scénaristes, dans deux ateliers, l'un écrivant les intrigues (résumées dans les "séquenciers") et l'autre rédigeant les dialogues, deux réalisateurs (l'un pour le tournage en studio, soit 2/3 des scènes, et l'autre pour le tournage des décors extérieurs), une centaine de techniciens et une trentaine de comédiens. Organisée sur le mode du flux, la production est continue tout au long de l'année, les épisodes sont écrits trois mois avant la diffusion, et filmés avec six semaines d'avance. Enfin, le feuilleton se distingue en étant un succès d'audience de longue durée avec 4 millions de téléspectateurs par soir en 2013.

3/ Il ne s'agit pas de professions au sens le plus strict du terme (Freidson, 1994), par commodité, nous utiliserons malgré tout le terme pour étudier ces métiers.

4/ « Territoire professionnel » est la manière dont Florent Champy traduit le terme de « juridiction » utilisé par Abbott, car en français le mot « juridiction » renvoie à des domaines de compétences définis de manière officielle (Champy, 2009 p. 79).

5/ La notion de « territoire professionnel » est en continuité avec celle de « mandat » proposée par Hughes (Champy, 2009 p. 177).

6/ Avec 122 heures annuelles de fiction pour Telfrance, soit $18 \%$ du volume total commandé par les chaînes nationales gratuites, et 117 heures pour Rendez-vous Production soit 17,2 \% du volume total (CNC, 2013 p. 29). 
L'organisation du travail sur le feuilleton redistribue en effet les tâches entre les différents professionnels impliqués, remettant en question les hiérarchies usuelles du champ de production audiovisuelle fortement marqué par la référence au cinéma (Darré, 2000). PBLV se singularise par la centralité du scénario dans le processus de production et par l'institutionnalisation d'une division intensive du travail d'écriture. Alors qu'au cinéma, la notion d'auteur renvoie souvent en France au réalisateur du film depuis la Nouvelle Vague (Mary, 2006), les « auteurs» désignent ici deux groupes de scénaristes chargés d'écrire le séquencier (la version résumée des séquences des épisodes), et les dialogues du feuilleton. Les auteurs du séquencier inventent les intrigues de la semaine d'épisodes, les dialoguistes transforment ces intrigues en répliques pour les personnages. Les réalisateurs sont chargés ensuite de mettre en image ces dialogues, en suivant l'esthétique du feuilleton. Les scénaristes prennent ainsi un rôle important dans la chaîne de production, quand les réalisateurs sont réduits à un rôle plus technique. Les contraintes imposées aux auteurs par la rationalisation de leur travail amènent à redéfinir leur périmètre d'activités, ce qui est source de tensions entre auteurs, du séquencier et des dialogues, mais également entre professionnels de l'écriture et de la réalisation.

Ces rivalités portent sur l'autonomie professionnelle, le degré de contrôle sur le travail, tous voulant maintenir les limites de leur territoire professionnel dans un cadre de production, certes rationalisé, mais encore travaillé par des incertitudes autour de la place de chacun. Ces tensions dans la chaine de fabrication correspondent également aux inégales ressources possédées par les différents intervenants ${ }^{7}$.

Pour étudier le travail de production de fiction télévisuelle, l'enquête s'est fondée sur une ethnographie du processus de fabrication du feuilleton PBLV, menée d'octobre 2008 à août 2010 avec plusieurs séjours d'observations des différentes étapes de la fabrication de l'écriture au tournage. Ce volet d'observation a été associé à une cinquantaine d'entretiens avec les agents de la fabrication du feuilleton, pour moitié avec les scénaristes du feuilleton, et pour moitié avec les autres intervenants du processus de production : producteur, responsables de l'écriture et de la réalisation, réalisateurs, assistants-réalisateurs et chargés du programme au sein de France 3. Pour objectiver les carrières des scénaristes, une base de données a été réalisée renseignant les trajectoires professionnelles de ceux engagés sur le feuilleton de 2004 à 2010.

$7 /$ Je remercie Céline Bessière, Émilie Biland, Pascal Marichalar et Audrey Mariette pour leurs relectures et leurs remarques. 


\section{L'ÉCRITURE EN MORCEAUX. UN MODĖLE INDUSTRIEL DE PRODUCTIONDE FICTION?}

Avant de revenir sur les tensions créées par la rationalisation du travail mise en place, il est nécessaire de s'arrêter sur ses spécificités. Le processus créatif, fortement morcelé et contrôlé, se rapproche en partie des modes de production des soap opera aux États-Unis (Allen, 1988). La production à flux tendu impose ainsi une division poussée du travail.

\section{- Les contraintes de la production d'un flux de fiction}

Rapporté aux budgets de formats plus nobles de la fiction télévisée, le budget de production de PBLV n'est pas si important. Pour produire 260 épisodes, la somme allouée aux producteurs était au moment de l'enquête (de 2007 à 2010) de 27 à 30 millions d'euros, soit un budget moyen par épisode de 80000 euros, et un coût horaire de 160000 euros. À titre de comparaison, le CNC estime en 2013 le coût moyen horaire de la fiction à la télévision à 1469000 euros pour les téléfilms unitaires de 90 minutes, et à 280000 euros pour les séries de 26 minutes $^{8}$. Outre ces contraintes économiques, la contrainte principale de fabrication est temporelle: la production en flux tendu ne peut être interrompue ou prendre du retard. Le flux impose une forme d'urgence à la fabrication, comme l'explique Jean ${ }^{9}, 45$ ans, auteur du séquencier depuis deux ans, issu du CEEA, école de formation au scénario télévisé ${ }^{10}$ : «En fait, la première particularité de l'écriture sur Plus Belle La Vie par rapport aux autres écritures de fiction, c'est le rythme. Ça va extrêmement vite». L'étape de l'écriture est ainsi rationalisée pour répondre à l'exigence de produire un flot ininterrompu d'épisodes. $\mathrm{Au}$ niveau du tournage, l'organisation du travail est proche du modèle cinématographique (Séroussi, 2006), tout en l'intensifiant : 5 épisodes sont filmés par semaine. Face aux normes du cinéma, où trois minutes utiles de film sont tournées par jour, le tournage enregistre environ 20 minutes de film par jour, selon un rythme très intensif classique dans ce type de format (Pasquier, 2008). Les réalisateurs qui se succèdent chaque semaine dans les studios et en extérieur, sont supervisés par un directeur artistique, et sont tenus

8/ Ainsi Canal + a produit en 2013, 6 heures de 26 minutes (la série Kaboul Kitchen) pour un budget de 5,8 millions d'euros soit un apport horaire de 958300 euros.

9/ Entretien réalisé en septembre 2010. Par souci d'anonymisation, tous les prénoms et noms des enquêtés ont été modifiés.

10/ CEEA : Conservatoire européen d'écriture audiovisuelle. 
de respecter les codes visuels du feuilleton. Ils acceptent en définitive un rôle de "super technicien», selon les termes du directeur de production, dans une division du travail qui met au centre l'étape d'écriture du scénario.

Contraints par le temps, les professionnels impliqués dans la fabrication sont fortement encadrés dans l'exécution de leurs tâches. Garants du respect des délais, à chaque étape, des « chefs de poste » s'assurent de l'uniformité des épisodes fabriqués. Un directeur d'écriture orchestre le travail des deux équipes de scénaristes. À l'étape du tournage, un directeur artistique supervise la réalisation dans son ensemble (de la construction de nouveaux décors aux choix des costumes), tandis qu'un directeur d'acteurs est chargé d'encadrer le jeu des comédiens. Enfin, un directeur de la postproduction supervise l'étape du montage. Ces intervenants, les plus en contact avec le producteur et la chaîne, sont chargés du rappel des contraintes économiques et aussi de l'attention au public. Lors de l'enquête, les hommes occupant ces postes étaient présents depuis les premiers épisodes, et avaient ainsi défini les principes narratifs et visuels de PBLV. Cette division des tâches correspond également à des modalités d'engagement différenciées sur le feuilleton. La plupart des professionnels sont employés de manière temporaire (pour des contrats de courte durée), mais récurrente (ils collaborent régulièrement à la fabrication). Lors de l'enquête, les scénaristes du séquencier sont engagés plusieurs semaines d'affilée, tandis que les dialoguistes travaillent chacun une ou deux semaines par mois. Une partie des techniciens n'est embauchée que pour le temps du tournage et de la préparation de 5 épisodes, d'autres pour des durées d'un mois environ. À l'exception des chefs de poste et des scénaristes (rémunérés par les droits d'auteurs et de diffusion), la règle est donc l'intermittence ${ }^{11}$. Les chefs de poste, embauchés sur une plus longue durée, encadrent les personnels de passage, tandis que, comme nous le verrons, la durée d'engagement des scénaristes les différencie aussi des autres intervenants.

La production de fiction audiovisuelle repose enfin sur la forte intervention non seulement de la chaîne, commanditaire de l'œuvre, mais aussi du producteur qui met en ouvre la fabrication (Dagnaud, 2006 ; Brigaud-Robert, 2011). Les producteurs et le diffuseur interviennent à chaque étape, tant sur les décisions de production que sur les contenus. Cette supervision commence avec l'organisation d'un séminaire annuel, réunissant les producteurs et les chefs de

11/ Une partie des techniciens du tournage employés par France 3 Provence-Alpes-Côte-d'Azur travaillent cependant très régulièrement pour le feuilleton, voire en permanence. 
poste pour décider des grandes lignes de l'évolution du feuilleton. Ils valident ensuite le séquencier, les dialogues, le casting et enfin le montage. Ce fort contrôle du producteur sur le contenu, constaté dans la production des soap operas (Cantor, Pingree, 1983, p. 52) était également une des caractéristiques des studios de cinéma à Hollywood des années 1940 (Powdermaker, 1950). Le contrôle ne pèse pas cependant de la même manière sur tous, certains intervenants pouvant avoir plus de maîtrise que d'autres sur leur travail. La chaîne de coopération repose sur une élaboration collective où personne n'a la mainmise sur tout le processus de production.

La chaîne de coopération repose sur une élaboration collective où personne n'a la mainmise sur tout le processus de production.

\section{Une intense division du travail d'écriture}

La rationalisation du processus morcelle donc le travail d'écriture pour répondre aux contraintes de la production d'un flux d'épisodes. Réalisée à Paris, l'écriture du scénario est divisée en deux étapes, répartie entre une quinzaine d'intervenants. Un premier groupe de huit auteurs réalise un résumé des séquences composant les 5 épisodes d'une semaine de diffusion (le séquencier), tandis qu'un second groupe d'auteurs écrit les dialogues. L'atelier séquencier est dirigé par le directeur d'écriture, impliqué dans l'écriture des intrigues principales, et secondé par un chef de l'atelier séquencier. Certaines fonctions de l'atelier sont tournantes: chaque semaine, un auteur est responsable de la version définitive du séquencier. Cet « auteur référent » est chargé de répondre aux questions des dialoguistes lors de la réunion hebdomadaire de coordination entre les deux étapes. Ensuite, un second groupe d'auteurs, composé de 5 dialoguistes, développe une première version dialoguée du scénario, la « V1». Ils sont encadrés par deux chefs de l'atelier dialogues, chargés d'harmoniser et de produire la version finale des dialogues des 5 épisodes de la semaine.

La division du travail a connu des transformations suite aux résultats d'audience décevants lors du lancement du feuilleton: avec l'arrivée d'un nouveau directeur d'écriture, l'étape du synopsis a été supprimée dans un souci d'efficacité. Ce système d'écriture reprend des traits présents dans de nombreuses fictions audiovisuelles. La division en étapes (synopsis, séquencier, continuité dialoguée) - mise en place à Hollywood - est classique dans l'écriture d'un scénario et a constitué un moyen (pour les producteurs et commanditaires) de se prémunir contre les aléas de la production et de contrôler la qualité du travail fourni (Caves, 2000). L'écriture en groupe avec des ateliers est en outre courante aux États-Unis (Newman, Levine, 2012), et pour les soap opera (Allen, 1988). En France, elle a été 
mise en place pour d'autres feuilletons quotidiens ${ }^{12}$. L'écriture collective est répandue pour les séries dans lesquels les épisodes ne sont pas indépendants, lorsque les intrigues courant sur plusieurs semaines nécessitent une coordination entre les scénaristes. Cela ne concerne cependant encore qu'une minorité des productions françaises. Selon la SACD, 13,7\% des commandes d'écriture de 52 minutes en 2011, impliquaient des ateliers d'écriture de 5 à 11 coauteurs (SACD, 2012). PBLV se distingue par la fréquence des ateliers, qui ont lieu deux jours par semaine mais aussi par le nombre de scénaristes impliqués dans l'écriture (15). Par sa régularité, sa longévité, et le nombre d'auteurs, l'organisation de l'écriture à PBLV systématise ainsi une division du travail existant sur d'autres formats. En effet, les fonctions décrites existent dans d'autres séries, mais elles ne font pas l'objet de spécialisation de long terme de la part des scénaristes, à part à $P B L V$ en raison de la récurrence des engagements et de la longévité du feuilleton. Tous les auteurs rencontrés collaboraient ainsi au feuilleton depuis au moins 6 mois, et pour certains depuis les débuts du feuilleton.

Cette organisation du travail redécoupe ainsi les territoires professionnels des scénaristes.

Cette organisation du travail redécoupe ainsi les territoires professionnels des scénaristes : par son caractère routinisé, elle crée des spécialisations au sein de la même profession, différenciant les auteurs des histoires et ceux des dialogues, suscitant des tensions autour des territoires professionnels usuels.

\section{CRÉATION COLLECTIVE ET RÉAPPROPRIATIONS DU PROCESSUS D'ÉCRITURE}

Le caractère collectif de l'écriture conduit chaque auteur à redéfinir son travail, et à apprendre à s'intégrer dans le groupe. La division de l'écriture en deux étapes crée deux groupes de professionnels avec des perspectives qui s'opposent parfois sur la fabrication, spécialisant les uns du côté des «histoires » et les autres du côté de la psychologie des personnages, chaque groupe défendant alors son « territoire ». Les oppositions entre les différentes étapes du scénario recoupent par ailleurs des inégalités de genre et des différences de carrière et de formation entre scénaristes.

12/ Cap des Pins (France 2, 1997, 281 épisodes), ou Paris 16e (M6, mars 2009, 80 épisodes), Seconde Chance (TF1, septembre 2008, 180 épisodes), 5 Socurs (France 2, janvier 2008, 108 épisodes), Pas de secrets entre nous (M6, juin 2008, 80 épisodes). 


\section{Se fondre dans le collectif}

La première tâche pour les auteurs est d'apprendre à écrire collectivement, en particulier pour l'atelier séquencier. Le travail des séquenceurs est soumis au contrôle du groupe qui donne son avis à plusieurs reprises sur les intrigues. Tenant compte de cette capacité à travailler collectivement, le recrutement se fait par cooptation, après un stage d'un mois. Les auteurs apprennent en même temps à respecter les contraintes de fabrication et à se fondre dans le groupe. Ils soulignent les avantages de cette dimension collective, qui leur permet de se reposer sur leurs collègues pour pallier les pannes d'écriture, comme l'explique Joëlle ${ }^{13}$, âgée de 49 ans, travaillant au séquencier depuis quatre ans, issue d'une école de cinéma :

\section{Enquêteur: Et qu'est-ce qui se passe quand vous n'avez pas les idées?}

Joëlle : «Bah, il y a quelqu'un d'autre qui en a! De toute façon, il n’y a pas le choix.»

L'écriture collective permet de répondre aux impératifs de production, tout en assurant une forme de contrôle par les séquenceurs eux-mêmes du respect des délais. Elle reste marquée par le poids de la hiérarchie. Yves ${ }^{14}$, le directeur d'écriture, résume : "C'est collectif. Moi, je dirige tout ». Lors des réunions, c'est lui qui tranche, parfois contre l'avis des auteurs.

\section{Observation réunion séquencier, octobre 2008 :}

«En octobre 2008, Claire, scénariste de 35 ans, diplômée du CEEA présente sur le feuilleton depuis 2 ans, négocie pour que son idée d'intrigue comique soit conservée. Cette intrigue implique le personnage d'une indienne proposant des soins ayurvédiques: un jeune adolescent pense que cette jeune femme qui reçoit des hommes dans sa chambre d'hôtel toute la journée est une prostituée et que son propre père est un des clients. Dans sa version, il est rapidement détrompé. Yves, le directeur d'écriture, insiste pour garder le quiproquo un peu plus longtemps. Il compare sa propre idée avec une scène des 400 Coups de Truffaut. Le lendemain, la jeune scénariste essaie de ne pas suivre la proposition d'Yves qu'elle trouve moins drôle. Lors du week-end, les corrections apportées par le directeur d'écriture réorientent l'intrigue dans le sens qu'il avait proposé. Le lundi matin, Joëlle commente : «c'est même pas la peine de discuter, c'est son idée. »

Le caractère collectif et hiérarchisé de l'écriture est parfois source de frustrations pour des auteurs. L'atelier ne suit pas pourtant le modèle de certains soap opera où un auteur en chef définit des lignes

13/ Entretien réalisé en octobre 2009. Tous les prénoms des personnes rencontrées ont été modifiés pour garantir leur anonymat.

14/ Réalisé en septembre 2008. 
directrices exécutées par des scénaristes sous son autorité (Allen, 1988). Il se rapproche davantage de l'atelier du peintre, le directeur d'écriture donnant les grandes lignes, et participant à l'écriture de la partie la plus importante, l'arche A, comme le maitre s'occupant des parties les plus valorisées du tableau, alors que ses apprentis peignent le fond de la toile (Baxandall, 1985). Cependant la signature n'est pas attribuée à une seule personne: le directeur d'écriture est mentionné séparément au générique (de début et de fin) de même que le chef de l'atelier séquencier, les autres apparaissent sous forme de liste comme auteurs du séquencier (dans le seul générique de début). La structure hiérarchique de l'atelier se traduit dans les modes de rémunération. Les scénaristes sont rémunérés avant la diffusion par un à-valoir touché pour chaque semaine d'écriture, puis en droits de diffusion. La part de l'à-valoir comme des droits de diffusion est dégressive selon l'ancienneté des différents auteurs. De plus, le rôle d'auteur référent amène une rémunération supplémentaire, et constitue une forme de promotion après un an d'exercice environ.

Ces hiérarchies sont structurées par l'ancienneté, comme dans les équipes de scénaristes des séries télévisées américaines (Caldwell, 2009 p. 227). Lors de l'enquête, sur 13 séquenceurs travaillant sur le feuilleton, on dénombrait sept hommes et six femmes, en comptant le directeur d'écriture. Deux autres étaient présents depuis les premiers épisodes, et quatre d'entre eux écrivaient pour le feuilleton depuis plus de trois ans. Ce sont ceux qui ont le plus d'expérience qui prennent le plus la parole lors des réunions, comme Sofia, présente depuis les débuts, âgée d'une quarantaine d'années et dotée d'une longue expérience, ou encore Mathieu, présent depuis deux ans et ayant fréquenté la formation en scénario de la FEMIS.

Au-delà du cadre collectif, des rapports de force construisent la distribution des tâches au sein du collectif des séquenceurs tout en restant limités en raison du caractère tournant des responsabilités et des fonctions. Habitués dans leurs autres projets à être le principal auteur ou à coopérer avec un plus petit nombre d'auteurs ${ }^{15}$, ils doivent apprendre à écrire avec les autres, et à déléguer une partie des tâches à d'autres scénaristes.

15/ En 2007, le nombre de coauteurs moyens pour les séries télévisées en 2003-2004 était de 3,83 (dont le réalisateur, reconnu par la SACD comme auteur de l'œuvre, et touchant aussi des droits de diffusion) (Benhamou, Peltier, 2007, p. 3). 


\section{L'histoire ou la psychologie : deux perspectives différentes sur le scénario}

C'est surtout entre les équipes des dialoguistes et des séquenceurs que des tensions ont été observées autour des territoires professionnels. Cela provient d'abord de leurs places respectives dans le processus de fabrication qui créent des rapports au scénario différents. Les séquenceurs visent à produire la structure des intrigues, à travers l'écriture de nombreux rebondissements. Chaque semaine, ils doivent écrire 85 séquences, réparties selon plusieurs types d'intrigues (ou « arches ») : des arches longues A, de type policier d'une durée de 40 épisodes, et occupant 8 séquences de chaque épisode ; des arches B traitant de «problèmes de société » de 10 à 20 épisodes, représentant 6 séquences dans chaque épisode; et enfin les intrigues $C$ de comédie s'étendant sur quatre séquences, d'un à deux épisodes. Le rôle des séquenceurs est de fournir des histoires en grand nombre, et de les raconter dans l'économie matérielle et narrative de PBLV. Joëlle explique : « Le jeudi quand on arrive, il faut avoir 40000 idées à la minute parce qu'autrement, il y a rien ». Pour les dialoguistes, il s'agit de développer les séquenciers en versions dialoguées correspondant à 22 minutes de film. Cela conduit à des différences de perspective entre auteurs du séquencier et des dialogues, comme l'explique Serge ${ }^{16}$, chef de l'atelier séquencier, issu de la FEMIS ${ }^{17}$, école nationale supérieure de cinéma, à $P B L V$ depuis quatre ans.

\footnotetext{
Serge: "C'est vrai que nous, on est dans une logique dramaturgique, donc de mécanique narrative, et eux, ils sont beaucoup plus sur la psychologie. Et donc c'est vrai que parfois, il y a des séquences où nous, on fait des trucs un petit peu en force. Et eux derrière, ils disent : "On va vachement avoir du mal à mettre tel personnage, à le faire entrer dans la scène à tel endroit psychologique, et le faire sortir comme ça, on ne va pas arriver à le tordre" ».
}

Ces perspectives sont liées à leur façon d'intervenir sur le scénario, comme le résume un dialoguiste ${ }^{18}$, issu de la FEMIS, âgé de 39 ans, présent depuis un an sur la série:

Charles: «Je vois bien en lisant leur séquencier qu'ils partent très très loin. Ils s'autorisent à peu près tout, enfin ils s'autorisent pas mal de choses quand même. Donc ils ont une plus grande liberté que nous aux dialogues, pour le coup. (...) Nathalie (responsable des dialogues) elle disait

16/ Entretien réalisé en juin 2009.

17/ Tous les enquêtés l'ont fréquentée en filière scénario.

18/ Entretien réalisé en octobre 2009 
un truc très très juste: "Au séquencier, ils peuvent être au second degré, quand ils travaillent, quand ils écrivent, alors que nous, on peut être que premier degré." »

Les séquenceurs mettent en avant la dimension de jeu de leur travail, Serge comparant l'atelier séquencier à « un joli train électrique », appréciant de placer les personnages dans des situations extrêmes, ou incongrues comme de faire de Wanda, septuagénaire, une opératrice de téléphone rose ${ }^{19}$, ou d'imaginer que Franck ${ }^{20}$, électricien, compagnon de Blanche et souvent absent, est un fait un membre de la DGSE. Par contraste, le travail des dialoguistes nécessite d'avoir des qualités d'empathie, de se mettre à la place des personnages, «de les sentir», comme le dit Chloé, dialoguiste ${ }^{21}$ de 27 ans, depuis un an sur le feuilleton, issue d'une école de cinéma du sud de la France. Ceux-ci questionnent alors les procédés narratifs utilisés par les séquenceurs, qui les amènent à faire changer rapidement d'opinion ou d'humeur un personnage.

Ces différences de perspectives sont sensibles lors des « réunions dialogues », moment de passage de relais entre les deux étapes, qui rassemblent l'auteur référent du séquencier, les 5 auteurs des dialogues, un chef de l'atelier dialogue, le directeur d'écriture, le chargé de production et la coordinatrice d'écriture. Lors de ces réunions, les questions des dialoguistes portent souvent sur la cohérence des personnages.

Réunion dialogues, lundi 15 décembre 2008 :

Épisode 1176, scène 14 : Céline et Virginie cherchent à avoir un enfant, Thomas a accepté d'être le père. Les séquenceurs ont imaginé que les futurs grands-pères de cet enfant non encore conçu perdent le sens des réalités en cherchant déjà un logement près de leur petit-enfant. Céline et Virginie s'en plaignent à Thomas, qui leur propose à son tour d'habiter chez elles, à leur consternation. Cela conduit à un comique de répétition. Claire est l'auteur référent de la semaine. Josiane, Christiane et Marie, dialoguistes expérimentées, âgées de 45 à 60 ans, travaillent depuis au moins quatre ans sur la série.

Claire : «La 14 commence par Céline et Virginie qui reviennent sur ce qui s'est passé. »

Marie à Christiane : "Tu crois que Thomas propose ça ? »

Christiane: "C'est pas Thomas, ça. C'est un mec vachement fin.»

Josiane: «Un peu mou comme séquence, il y a rien là dans la séquence. »

19/ Intrigue diffusée lors de la saison 5 en juin 2009.

20/ Saison 6 diffusée au printemps 2010.

21/ Entretien réalisé en mai 2010. 
Les dialoguistes remettent en question l'intention comique pour rappeler la logique des personnages: Thomas, plein de tact, ne proposerait pas quelque chose d'aussi déplacé. Elles reprochent également aux séquenceurs le caractère «vide» ou «mou» des séquences, c'est à dire leur absence de matière narrative. Ces questions sont dictées par l'exigence de leur tâche: produire un certain nombre de répliques pour respecter la durée des épisodes.

La division des tâches séparant l'élaboration des histoires de l'écriture des dialogues conduit à des tensions qui se cristallisent autour de la maîtrise de l'histoire des séquenceurs, et donc du contrôle par les dialoguistes de leur territoire professionnel. Les auteurs des dialogues sont parfois laissés dans l'ignorance de la suite des intrigues. Lors d'une observation, ceux-ci se plaignent de ne pas être informés du dénouement des histoires, y voyant une forme de mépris de la part des responsables de l'écriture.

\section{Observation juin 2010 :}

Avant la réunion dialogues, prévue à $14 \mathrm{~h} 30$, les dialoguistes discutent des séquenciers et sont assez sceptiques. L'une d'entre eux juge l'intrigue « abracadabrantesque ». Ils ne comprennent pas sur quoi vont déboucher les intrigues B de la semaine: "Parfois les B, il n'y a pas d'enjeu », commente Christiane, dialoguiste expérimentée, présente depuis les débuts du feuilleton. C'est en effet la politique des chefs de l'atelier dialogue de ne pas leur dire la suite des intrigues. Charles explique qu'il trouvait ça bien au début pour garder «l'esprit frais», mais qu'il a changé d'avis. Christiane dit qu'ils sont des scénaristes, qu'ils ont écrit des polars, qu'ils ne sont pas débiles et qu'ils savent ne pas dévoiler les choses. Ce refus de les informer fait penser aux dialoguistes que les séquenceurs ne savent pas où ils vont.

Les dialoguistes se sentent dépossédés par leur ignorance de la suite des intrigues, sans maîtrise du processus d'écriture. Certains ont d'ailleurs exprimé le souhait d'assister à des réunions de l'atelier séquencier et de participer à l'écriture des intrigues. Une tentative au printemps 2010 de coécrire des arches A en appariant un séquenceur et un dialoguiste a tourné court, créant de la déception chez les auteurs des dialogues s'étant prêtés à l'exercice. Le mode de division des tâches du feuilleton crée ainsi des frustrations, en séparant des tâches que les scénaristes exercent souvent conjointement, et en

Le mode de division des tâches du feuilleton crée ainsi des frustrations, en séparant des tâches que les scénaristes exercent souvent conjointement. 


\section{Les dialoguistes et les séquenceurs: des carrières similaires, des métiers différents}

Ces tensions autour de la définition de leur territoire professionnel sont liées aux modalités d'organisation de la fabrication, mais aussi aux similarités et aux différences entre les carrières de ces professionnels.

Cette répartition des tâches correspond d'abord à une forme de division sexuelle du travail ${ }^{22}$ (Kergoat, 2010). Même si la population des auteurs de PBLV est plus féminisée que la catégorie des auteurs de l'audiovisuel ${ }^{23}$ (Gouyon, 2011, p. 6) avec 45 femmes et 42 hommes ayant au total collaboré au feuilleton de 2004 à $2011^{24}$, cette relative féminisation est à nuancer. Sur le total des auteurs réguliers (ayant travaillé au moins plusieurs mois), la proportion de femmes diminue: 22 hommes et 21 femmes. Cette féminisation ne s'étend pas non plus aux postes d'encadrement, à l'écriture comme sur le tournage, occupés pendant l'enquête par des hommes de plus de 45 ans, à l'exception de la coresponsable de l'atelier dialogues. C'est ce que souligne Marie ${ }^{25}$, dialoguiste issue de la FEMIS en scénario et âgée d'une quarantaine d'années: "Dans l'équipe, il faut quand même voir qu'aux postes de pouvoir et cadres, il y a que des hommes, c'est pas pour rien, à part Nathalie ». De même, dans la distribution des tâches entre scénaristes à Hollywood, les femmes se voient moins souvent confier des postes à responsabilité (Bielby, Bielby, 1996).

Cette répartition verticale des postes recoupe une autre division des tâches selon le genre. Sur les 43 auteurs réguliers, on compte 13 hommes et 8 femmes au séquencier, et 13 femmes et 9 hommes aux dialogues. Cela correspond à des modalités de recrutement différenciées entre les deux ateliers qui ne valorisent pas les mêmes qualités. À l'atelier séquencier, il s'agit de montrer son intégration au groupe, lors d'un stage décrit comme éprouvant par de jeunes auteurs tel Mathieu ${ }^{26}, 32$ ans, entré sur le feuilleton alors qu'il était

22/ Organisée selon Danièle Kergoat selon « un principe de séparation » (il y a des travaux d'hommes et des travaux de femmes) et un «principe hiérarchique » (un travail d'homme «vaut » plus qu'un travail de femme) (Kergoat, 2010 p. 65).

23/ Les femmes représentent $24 \%$ des auteurs de la SACD ayant touché des droits pour une œuvre audiovisuelle en 2008 (Gouyon, 2011).

24/ La base de données a été réalisée à partir de la collecte des CV sur les sites des agents artistiques, des lieux de formation ou en dernier recours sur le site Internet Movie Data Base. Un grand nombre de scénaristes travaille pour un mois de stage sans être embauché. J'ai retenu ceux ayant collaboré pour des durées plus longues.

25/ Entretien réalisé en février 2010.

26/ Entretien réalisé en juin 2010. 
sans engagement depuis deux ans depuis sa sortie de la FEMIS. Il décrit le difficile équilibre à maintenir lors du stage, entre mise en avant de ses idées et attitude de modestie, racontant qu'il a parfois eu peur de paraître arrogant. À l'inverse, des jeunes femmes ont ressenti comme difficile le fait de s'imposer face au groupe, et ont été réorientées par le directeur d'écriture de l'époque vers les dialogues. C'est le cas de Julie ${ }^{27}, 37$ ans, issue elle aussi de la FEMIS :

\begin{abstract}
Julie: «J'avais un peu de mal à trouver ma place parce c'est des réunions, il faut sortir plein d'idées, il faut s'imposer dans le groupe, et voilà. $\mathrm{Au}$ bout de six mois, le directeur d'écriture m'a dit que je serais sans doute mieux aux dialogues. Et effectivement, ça a été beaucoup mieux aux dialogues. Puisqu'on est plus solitaire aussi. (...) [Au séquencier] quand on présente les idées, il faut s'imposer, il faut défendre son bifteck, il faut dire que c'est vachement bien. Et moi, ça j'ai du mal à le faire. Il faut vraiment avoir un tempérament un peu de battant. »
\end{abstract}

Comme Mathieu, Julie décrit le travail du séquencier comme nécessitant de se mettre en avant face au groupe, en opposition avec l'aspect solitaire des dialogues. Son expérience rejoint celle de Chloé, autre jeune auteure orientée vers les dialogues en raison de son attitude trop en retrait. Dans la description des postes, comme dans le discours opposant les « histoires » et « la psychologie » se retrouvent des représentations genrées des deux fonctions, associant aux séquenceurs des qualités « masculines » (l'action, l'esprit de compétition) plus valorisées, et aux dialoguistes des qualités « féminines » (instinct, sensibilité, empathie) moins valorisées, car réputées être acquises individuellement plus que par une formation (Kergoat, 2010 p. 66). Cette association entre femmes et sentiments a également été constatée à Hollywood où les femmes sont plus souvent auteures de feuilletons sentimentaux (Bielby, Bielby, 1996). Cette dimension genrée des fonctions constitue également une hiérarchisation, les dialoguistes jouissant d'une plus grande solitude mais également d'une moindre autonomie dans leur travail. Le travail sur la « structure » des scénarios est ainsi plus valorisé que celui sur les dialogues (Pasquier, 1995, p. 84).

Les tensions entre séquenceurs et dialoguistes peuvent alors s'expliquer par l'inégal pouvoir décisionnel des deux fonctions, en décalage avec la similarité de leurs formations et carrières. La différenciation entre auteurs des dialogues et des histoires existe sur d'autres séries, sans faire pour autant l'objet d'une reconnaissance 
professionnelle ${ }^{28}$. Les contrats des scénaristes différencient l'arche narrative, le synopsis, le séquencier et la continuité dialoguée (SACD, 2012), mais ces différentes étapes peuvent être prises en charge par un seul auteur comme réparties entre plusieurs. Dans $70 \%$ des cas, les scénaristes du synopsis cumulent leur fonction avec celle de dialoguiste (Benhamou, Peltier, 2007, p. 3). Les scénaristes ont tous travaillé sur d'autres formats de fiction, à la télévision comme au cinéma, avant ou pendant leur passage à PBLV (pendant l'enquête, 9 des 12 auteurs du séquencier avaient des projets de long-métrage ou de séries parallèlement à leur participation au feuilleton). Si une partie des dialoguistes s'est spécialisée dans cette fonction, ils ont tous également signé des scénarios complets, tout comme les séquenceurs. Leurs cursus les ont formés à écrire des histoires comme des dialogues. Plus de la moitié ont fréquenté des écoles d'écriture de scénario, parfois très prestigieuses (comme la FEMIS, fréquentée par 10 des 43 auteurs lors de l'enquête, 5 dialoguistes et 5 séquenceurs). Issus pour la plupart de classe supérieure, les scénaristes rencontrés ont dans l'ensemble un niveau de diplôme élevé. Au-delà de ces similitudes, les deux groupes d'auteurs se différencient cependant par la dimension du genre, combinée avec leurs lieux de formations et les formes prises par leurs carrières. Les séquenceurs sont plus souvent passés par des formations spécialisées en scénario ou en cinéma (16 d'entre eux sur 21), et sont en moyenne plus diplômés que les dialoguistes ${ }^{29}$. En outre, les auteurs des dialogues sont globalement un peu plus âgés avec une moyenne d'âge de 48,5 ans contre 41,4 ans au séquencier, et ont pour une partie d'entre eux écrit sur des formes de fiction plus dévalorisées comme des sitcoms, des feuilletons quotidiens, ou encore des sketchs radiophoniques.

La rationalisation du processus d'écriture amène à diviser des tâches traditionnellement prises en charge par un même auteur, créant ainsi des rivalités entre dialoguistes et séquenceurs autour de la définition de leurs territoires professionnels. Cela s'articule à la place secondaire des dialogues dans l'écriture du scénario. Les tensions proviennent alors peut-être de l'acceptation par les dialoguistes de leur moindre autonomie. Cela correspond à une division sexuelle du travail, combinée à des différences de formation et de carrières entre auteurs des dialogues et des séquenciers. C'est aussi vis-à-vis de l'étape de la réalisation que ces conflits de définition se jouent entre les scénaristes dans leur ensemble et les responsables du tournage. Des concurrences autour de la définition des territoires

28/ Il existe une Guilde des scénaristes pour les auteurs de cinéma et de télévision, mais pas d'association professionnelle des dialoguistes.

29/ Ils sont ainsi 15 à disposer d'un niveau Bac +4 contre 9 dialoguistes. 
professionnels opposent ainsi l'étape de la préparation et du tournage à celle du scénario, autour de la place centrale occupée par ce dernier.

\section{- Le pouvoir de l'écriture}

Les tensions autour de la distribution des tâches entre écriture et tournage se cristallisent sur la question des didascalies. L'usage de ces indications scéniques révèle les tensions qui peuvent exister autour de la définition des territoires professionnels des scénaristes et de la direction artistique.

\section{- Les didascalies ou quand le scénario empiète sur la réalisation}

Le scénario doit, par les dialogues et avec le minimum de didascalies, fournir les informations nécessaires au tournage. Les versions dialoguées précisent un certain nombre d'éléments encore peu détaillés dans le séquencier, comme les lieux en extérieur, les déplacements d'un personnage dans la scène, l'état physique d'un personnage ou certains accessoires importants pour la narration. Avant la réunion dialogues, une réunion de production rassemble le producteur, le directeur d'écriture, les responsables des dialogues et l'auteur référent de la semaine pour amender le séquencier en fonction des remarques de la chaîne et des responsables du tournage sur la faisabilité des séquences. Ces aménagements sont transmis aux dialoguistes lors de la réunion dialogues chargés de les intégrer à la version dialoguée. Cependant la mise en scène en elle-même ne relève pas du territoire des scénaristes qui ne maîtrisent pas les contraintes spécifiques du tournage. Les dialoguistes se limitent donc dans leur usage des didascalies comme l'explique Charles, issu de la FEMIS, ayant écrit et réalisé des longs-métrages :

Charles: "C'est vrai que j'ai plutôt tendance à être un scénariste qui écrit beaucoup de didascalies, et qui pense beaucoup la mise en scène. Et pour cause, je voulais être réalisateur. Donc je pense vachement la mise en scène dans la séquence. Et ça influe complètement sur l'écriture. (...) [Sur PBLV], les didascalies sont réduites à la partie congrue. Donc on ne peut plus écrire que ce qui est vraiment essentiel à la compréhension de la séquence. (...) C'est pour pas qu'on empiète sur l'artistique et sur le plateau, et je le comprends. »

L'usage de ces indications scéniques délimite leurs champs de compétences, laissant la mise en scène à la direction artistique qui supervise la réalisation, et le jeu au directeur d'acteur. Les dialoguistes écrivent malgré tout des didascalies, précisant les sentiments d'un 
personnage, donnant des détails sur une action effectuée. Cela suscite souvent la dérision des membres de la direction artistique. Lors d'une réunion en février 2009, un membre de l'équipe de direction artistique s'exclame : «On s'en fout des didascalies, j’aimerais qu'ils n'écrivent absolument pas de didascalies ». Julien, l'adjoint du directeur artistique, ironise régulièrement sur les didascalies et les méconnaissances pratiques qu'elles révèlent de la part des scénaristes.

Réunion « accessoires », 10 février 2009:

Julien, directeur artistique adjoint, son assistant, ainsi que le réalisateur, le second assistant réalisateur, l'accessoiriste et le responsable des décors définissent l'usage des décors, les accessoires, et la mise en scène des scènes tournées en intérieur la semaine suivante. Julien a les scénarios devant lui et passe d'une séquence à l'autre.

Séquence 1182/08:

Guillaume est avec Florian dans le bureau de ce dernier... Florian est en train d'enfiler sa robe.

Florian : «Je n'ai pas beaucoup de temps, j'ai une audience au tribunal dans un quart d'heure.»

Julien, haussant les sourcils, pour marquer sa consternation : «Florian n'enfile pas sa robe, c'est une invention des scénaristes ». Il poursuit: «Car Florian dit: "j'ai une audience au tribunal dans un quart d'heure". Alors qu'il y est! »

Bernard : "C'est comme les didascalies. Les didascalies! "Véra regarde tristement partir Benoit et Raphaël". C'est une connerie, ça pour moi. Et si j'ai envie que Véra les regarde partir en colère, c'est MON problème! »

Enquêtrice: Donc vous ne suivez pas les didascalies?

Bernard : «Jamais, je ne les lis même pas! Et le premier conseil que je donne aux acteurs, les nouveaux comme les anciens, je leur dis, je les encourage, je les invite à ne pas lire les didascalies. Et moi, je ne les respecte pas!»

Les didascalies sont vécues

comme une

manière

d'empiéter sur son aire de

compétence et ses prérogatives.

Les didascalies sont vécues comme une manière d'empiéter sur son aire de compétence et ses prérogatives. Il livre aussi sa propre interprétation de la scène, se détachant de celle indiquée par le scénario. Le refus par la direction artistique et la direction d'acteur de suivre les didascalies leur permet ainsi de se laisser une marge de manœuvre dans l'interprétation du texte, et de mettre en avant leur connaissance pratique du jeu et du tournage.

La répartition des tâches entre le tournage et l'écriture se révèle aussi genrée. L'étape des dialogues est plus féminisée, tandis qu'à PBLV comme dans l'audiovisuel le tournage reste très masculin (Gaudy, 2008) : tous les responsables du tournage sont des hommes, ainsi que la plupart des réalisateurs (2 femmes sur les 10 rencontrés) et des assistant réalisateur. Les responsables du tournage semblent 
cependant s'opposer à tout le groupe des auteurs compris au masculin neutre, souvent sans différencier entre dialoguistes et séquenceurs. Si la dimension de genre est présente, elle s'articule aux différences et similitudes entre les formations et les carrières des professionnels du tournage et de l'écrit, et en particulier à la référence commune au cinéma et à la figure de l'auteur-réalisateur. D’après les entretiens réalisés, les réalisateurs mais aussi les assistants réalisateurs ont fréquenté des écoles de cinéma, le plus souvent moins prestigieuses que celles fréquentées par les scénaristes. Une recherche sur les CV des réalisateurs montre qu'aucun n'est passé par la FEMIS, par exemple. Le directeur artistique adjoint a commencé sa carrière comme régisseur sur des films de cinéma où il a acquis une expérience pratique, avant d'être second assistant réalisateur à $P B L V$. Le directeur d'acteur, âgé d'une cinquantaine d'années a une longue carrière au théâtre et au cinéma, et c'est aussi son expérience du jeu et de la mise en scène qu'il défend dans son dédain vis-à-vis des didascalies, rejouant ici la place dévalorisée de la télévision face au théâtre dans le champ de production culturelle. Ces rivalités professionnelles sont moins claires pour le directeur artistique, diplômé d'une maîtrise en histoire et ayant travaillé dans la communication d'entreprise.

Plus encore qu'entre les scénaristes, ces concurrences reposent sur la place de ces intervenants dans le processus de fabrication. Ce sont les responsables d'une étape de production (direction artistique et d'acteurs et dans une moindre mesure réalisateurs) qui s'opposent aux travailleurs de l'autre étape (et non à leurs responsables). Dans ces rivalités professionnelles, c'est le pouvoir des auteurs sur le feuilleton qui est source de tensions entre les différentes étapes.

Dans ces rivalités professionnelles, c'est le pouvoir des auteurs sur le feuilleton qui est source de tensions entre les différentes étapes.

\section{- Des auteurs puissants?}

La division du travail se traduit par un inégal pouvoir d'intervention et une inégale connaissance des intrigues, où réside, selon ses fabricants, une partie de la valeur du feuilleton ${ }^{30}$. Les dénouements des intrigues principales ne sont pas connus de tous, les dialoguistes ignorent parfois la suite des histoires, tandis que les comédiens ne sont pas toujours informés du devenir de leurs personnages. La plupart des techniciens mais aussi les assistants réalisateurs et les réalisateurs n'ont aucune vue sur les dénouements des intrigues. Par leur connaissance de l'intégralité des histoires, les membres de la 
direction artistique et de la direction d'acteurs sont les seuls sur le plateau à pouvoir prendre certaines décisions, comme de changer le décor d'une scène par exemple.

Cette maîtrise des histoires différencie les séquenceurs des autres intervenants. Par leur position même dans la chaîne de production, ils connaissent la fin des intrigues puisqu'ils en sont les auteurs. De plus, la production en flux ne permet pas de revenir en arrière pour corriger les défauts de l'étape précédente, plaçant les séquenceurs dans une position de pouvoir. La chaîne de fabrication conduit alors à « rattraper » le travail de l'étape précédente, créant des tensions entre les étapes. Cela se cristallise autour de la première étape, le séquencier. Mélanie $^{31}$, auteure du séquencier depuis un an, explique que la rapidité empêche un certain nombre de précisions à cette étape : « On n’a pas le temps d'être précis. Ça de toute façon, on sait que c'est aux dialogues d'arranger les choses, de préciser ». En effet il s'écoule trois semaines entre la première et la seconde version des dialogues, donnant du temps pour des reprises. Chloé, une dialoguiste qui a écrit des deuxièmes versions des dialogues souligne les nombreuses modifications qu'elle doit opérer en raison des défauts éventuels du séquencier :

Chloé : « On ne s'est pas rendu compte dans le séquencier que les personnages n'étaient pas très cohérents. C'est aussi une adaptation par rapport à la semaine d'avant, en fait, les deux semaines étaient un peu une redite l'une de l'autre, donc il fallait monter d'un cran. Souvent, c'est le séquencier qu'on corrige après coup. »

Ces corrections sont cependant à la marge, car les intrigues d'une semaine ne peuvent être réécrites dans leur intégralité. C'est l'organisation en flux qui crée les tensions entre les professionnels de chaque étape. Lors de mon séjour sur le plateau en février 2009, les membres de la direction artistique sont particulièrement remontés contre «les auteurs ». Les reproches sont liés à la prévision du tournage : la semaine de tournage en intérieur des épisodes prévoit plus de séquences que d'habitude, et nécessite le recours à des heures supplémentaires.

Observation dans les locaux de la production, février 2009

Un membre de la direction artistique explique à un réalisateur que le problème provient des séquenceurs : « Il y a des problèmes de structure. Donc ensuite on est obligé de couper des séquences pour rajouter des séquences. À l'artistique, on rajoute des trucs, sinon on a rien ». Il ajoute : «Il faut calmer les auteurs!» 
Les auteurs, en particulier ceux du séquencier, sont ainsi placés en position de pouvoir par leur intervention au début de la chaîne, alors que le flux de la production empêche qu'on revienne complètement sur les décisions prises. Ils ont paradoxalement une plus grande liberté et un pouvoir sur le contenu plus important que dans d'autres formats audiovisuels car leurs textes ne sont pas modifiés à maintes reprises en raison du rythme de production. Cela donne aux séquenceurs une place centrale dans le processus de fabrication, créant parfois des tensions avec le producteur. Celui-ci m'explique ${ }^{32}$ en mai 2010, qu'il cherche un moyen de responsabiliser les auteurs du séquencier et de contrôler davantage leur travail.

L'organisation de la fabrication donne ainsi une place importante au scénario, redéfinissant une partie des hiérarchies existantes dans le monde audiovisuel. Plus précisément, elle donne un grand pouvoir aux séquenceurs qui interviennent en premier dans le processus de production. C'est le contrôle plus grand de ces professionnels sur leur travail (même s'ils sont contraints par l'urgence) qui crée des concurrences avec d'autres professionnels, dont les territoires dépendent des tâches des séquenceurs.

L'organisation de la fabrication donne ainsi une place importante au scénario, redéfinissant une partie des hiérarchies existantes dans le monde audiovisuel

\section{Conclusion}

Les effets de l'industrialisation de la production sur le travail de scénaristes sont complexes. De même que les recherches sur le travail ouvrier ont pu mettre en évidence des espaces de résistance et d'autonomie relative des ouvriers au travail (Roy, 2006), les différents professionnels impliqués dans la fabrique du feuilleton ont des marges de manœuvres face aux contraintes de production. On observe également des inégalités entre eux dans la maitrise de leur travail. La rationalisation de la fabrication, si elle redéfinit les territoires professionnels des uns et des autres, ne conduit pas inéluctablement à la dépossession de ces travailleurs. Chaque groupe professionnel tente ainsi de défendre une forme d'autonomie dans le cadre fortement contraint d'une industrie culturelle.

La structure même de la division du travail sur le feuilleton conduit à des tensions entre les logiques professionnelles de chaque étape. La place du séquencier au début de la chaîne amène le reste des fabricants à devoir travailler à partir de cette matière première,

32/ Discussion informelle en mai 2010. Peu après est ajoutée une étape supplémentaire du séquencier une V3 pour avoir le temps de corriger les séquenciers. L'organisation du travail connaît ainsi des ajustements réguliers, j'ai décrit celle observée lors de l'enquête. 
ce qui crée des conflits liés à la distribution du pouvoir et de la responsabilité sur le feuilleton. C'est à la fois le contenu des tâches, qui accorde la primauté aux histoires dans la fabrique des épisodes, mais aussi la forme prise par l'organisation du travail, en flux, qui donnent aux auteurs du séquencier une plus grande maitrise sur leur travail relativement aux autres professionnels intervenant après eux dans la chaîne de production.

Cette inégale maîtrise des professionnels sur leur travail s'articule aux carrières des auteurs, mais relève également d'une forme de division sexuelle du travail, spécialisant les femmes dans les dialogues et plaçant des hommes aux postes d'encadrement. Les concurrences entre professionnels autour de leur territoire sont définies à la fois par la division du travail elle-même, mais aussi par les ressources des uns et des autres. Ces redistributions se réalisent selon les positions occupées dans le champ de production audiovisuelle, au profit de l'atelier plus masculin et plus diplômé. Par leurs formations, mais aussi leur place dans le processus de production, les scénaristes, et en particulier les séquenceurs, sont plus autonomes dans leur travail que d'autres intervenants. Ce sont des individus formés dans des univers artistiques, amenés à travailler dans un cadre industriel. Ces contradictions entre leurs ambitions initiales, leurs univers de référence et l'organisation du travail sur le feuilleton permettent en partie de comprendre les tensions dans la chaîne de fabrication, et de voir comment des rapports de force liés aux positions dans le champ de production culturelle jouent dans la chaîne de coopération. L'analyse du contenu du travail et de l'organisation de la production, en étant combinée à l'analyse des carrières des différents intervenants, permet d'expliquer les ressorts des rivalités professionnelles. Les tensions dans la distribution des tâches se traduisent ainsi dans les interactions quotidiennes de travail. Les concurrences entre professionnels pour préserver leur territoire s'apparentent à une volonté, pour ces travailleurs invisibles de la culture, de conserver une part de leur autonomie sur leur travail de création.

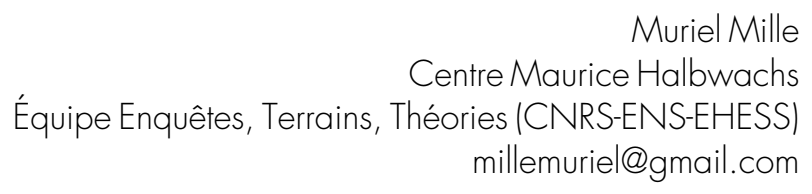




\section{RÉFÉRENCES BIBLIOGRAPHIQUES}

ABBOTT A., 1988

BACQUE M.-H. et al., 2014

ALLEN R. C., 1988

BANKS M., MAYER V., CALDWELL J. T., 2009

BAXANDALL M., 1985

BECKER H. S., 1988 [1984]

BENHAMOU F., PELTIER S., 2007 «Économies des droits d'auteur, III. La télévision »,

BIELBY D., BIELBY W., 1996

BOURDIEU P., 1992

BOURDIEU P., 1971

BRIGAUD-ROBERT, N., 2011

CALDWELLJ. T., 2009

CALDWELLJ. T., 2008

CANTOR M. G., PINGREE S., 1983

CANTOR M., 1971

CAVES R., 2000

CHAMPY F., 2009

CNC, 2014

CNC, 2013

CNC, 2009

DAGNAUD M., 2006

DARRE Y., 2000

ESQUENAZI J.-P., 2010

FREIDSON E., 1994 Culture Études, 6, p. 1-24.

The System of Professions. An Essay on the Division of Expert Labor, Chicago (Ill.) : The Chicago University Press.

«The Wire : l'Amérique sur écoute, Paris : La Découverte.

Speaking of Soap Operas, Chapel Hill (N. C.) : University of North Carolina Press.

Production studies. Cultural Studies of Media Industries, Londres : Routledge.

L'CEil du Quattrocento, Paris : Gallimard.

Les Mondes de l'art, Paris : Flammarion.

«Women and Men in Film : Gender Inequality among Writers in a Culture Industry », Gender and Society, 10 (3), p. 248-270.

Les Règles de l'art : genèse et structure du champ littéraire, Paris : Seuil.

« Le marché des biens symboliques », L’Année sociologique, 22 , p. $49-126$

Les Producteurs de télévision: socio-économie d'une profession, Vincennes: Presses universitaires de Vincennes.

« Both Sides of the Fence », in Banks M., Mayer V. Caldwell J. T. Production Studies. Cultural Studies of Media Industries, Londres : Routledge, p. 214-229.

Production Culture : Industrial Reflexivity and Critical Practice in Film and Television, Durham (N. C.) : Duke University Press.

The Soap Opera, Londres : Sage.

The Hollywood Television Producer, New York (N. Y.) : Basic Books.

Creative Indutries : Contracts Between Arts and Commerce, Cambridge (Mass.) : Harvard University Press.

La Sociologie des professions, Paris : PUF.

La Production audiovisuelle aidée en 2013, Paris : Les études du CNC.

« Les programmes audiovisuels », Les Dossiers du CNC, 328.

Les Chiffres-clés de la télévision hertzienne (1998-2007), Paris : Les études du CNC.

Les Artisans de l'imaginaire : comment la télévision fabrique la culture de masse, Paris : A. Colin.

Histoire sociale du cinéma français, Paris : La Découverte. Les Séries télévisées : l'avenir du cinéma ?, Paris : A. Colin.

« Pourquoi l'art ne peut pas être une profession », in Menger P.-M., Passeron J.-C., L'Art de la recherche. Essais en l'honneur de Raymonde Moulin, Paris : La documentation française, p. 119-135. 
GAUDY C., 2008

GITLIN T., 2000

GOUYON M., 2011

HESMONDHALGH D., 2002

HUGHES E. C., 1996

KERGOAT D., 2010

MARY P., 2006

MATHIEU L., 2013

NEWMAN M.Z., LEVINE E., 2012 Legitimating Television: Media Convergence and Cultural Status, Londres : Routledge.

NEWCOMB H., ALLEY R.S., 1983 The Producer's Medium, Oxford : Oxford University Press.

PASQUIER D., 1995

POWDERMAKER H., 1950

ROY D., 2006

SACD, 2012

SACD, 2009

" "Être une femme" sur un plateau de tournage ", Ethnologie française, 38 (1), p. 107-117.

Inside Prime Time, Berkeley (Calif.) : University of California Press.

« Diversité des carrières d'auteurs de l'audiovisuel et du spectacle vivant. Les auteurs de la SACD, 1997-2008 », Culture Chiffres, Paris : Ministère de la Culture.

The Cultural Industries, Londres : Sage.

Le Regard sociologique : essais choisis, Paris : Éditions de l'EHESS.

« Le rapport social de sexe. De la reproduction des rapports sociaux à leur subversion », in Bidet A., Les Rapports sociaux de sexe, Paris : PUF, p. 60-75.

La Nouvelle Vague et le cinéma d'auteur : Socio-analyse d'une révolution artistique, Paris : Seuil.

Columbo: La lutte des classes ce soir à la télé, Paris : Textuel.

Les Scénaristes et la télévision : approche sociologique, Paris : INA.

Hollywood, the Dream Factory : an Anthropologist Looks at the Movie-Makers, Boston (Mass.) : Little Brown and Company.

Un sociologue à l'usine : textes essentiels pour la sociologie du travail, Paris : La Découverte.

Observatoire permanent des contrats audiovisuels 2012, Paris : SACD.

Observatoire permanent des contrats audiovisuels 2009, Paris : SACD.

SEPULCHRE S., MAIGRET É., 2011 Décoder les séries télévisées, Paris : De Boeck.

SEROUSSI B., 2006

« La création au travail. Le film, produit d'un collectif », in Bidet A. Borzeix A., Sociologie du travail et activité, Toulouse : Octarès. 